\title{
Global VLBI Observations of M82
}

\author{
A. R. McDonald, T. W. B. Muxlow, A. Pedlar. \\ University of Manchester, Jodrell Bank Observatory, Lower Withington, \\ Macclesfield, Cheshire SK11 9DL, UK.
}

\section{A. Garrett}

Joint Institute for VLBI in Europe, Postbus 2, 7990 Dwingeloo, The Netherlands.

\section{K. A. Wills}

Department of Physics, University of Sheffield, Sheffield S3 7RH, UK.

\begin{abstract}
Images are presented of the starburst galaxy M82 from a 20station global VLBI experiment with an angular resolution of up to 3 mas. These images resolve several of the sources into a number of components and with future VLBI experiments the expansion of these components may be tracked on a $\sim 2$ year timescale. Expansion of one of the brightest remnants in M82 has already been measured on a 10 year timescale using the European VLBI Network (EVN) alone and an expansion rate of $10,000 \mathrm{~km} \mathrm{~s}^{-1}$ calculated. Furthermore, limits on the deceleration of this remnant have been deduced. Further VLBI experiments will be able to better constrain the deceleration of the remnants on a $\sim 10$ year timescale as they continue to interact with the interstellar medium in M82.
\end{abstract}

\section{Introduction}

M82 is generally considered to be the archetypal starburst galaxy. Far infrared and radio luminosities imply a high starformation rate and at a distance of 3.2 $\mathrm{Mpc}$ it lends itself well to study with radio interferometry on parsec scales.

The radio studies of M82 have revealed a population of $~ 50$ compact sources (Unger et al. 1984; Kronberg, Biermann \& Schwab 1985) which were originally tentatively identified as being either radio supernovae or young supernova remnants. Subsequent observations with MERLIN at a resolution of 50 mas $(0.75$ pc) resolved most of the compact sources and the parsec-scale shell-like structure observed in many of the objects confirmed them to be mainly young supernova remnants (Muxlow et al. 1994).

This paper reports on the latest 20-station global VLBI experiment at $\lambda 18 \mathrm{~cm}$ to concentrate on M82 which was observed in November, 1998 . Due to the decrease in surface brightness sensitivity with the increase in resolution provided by VLBI only two of the sources were well detected, the aforemen- 


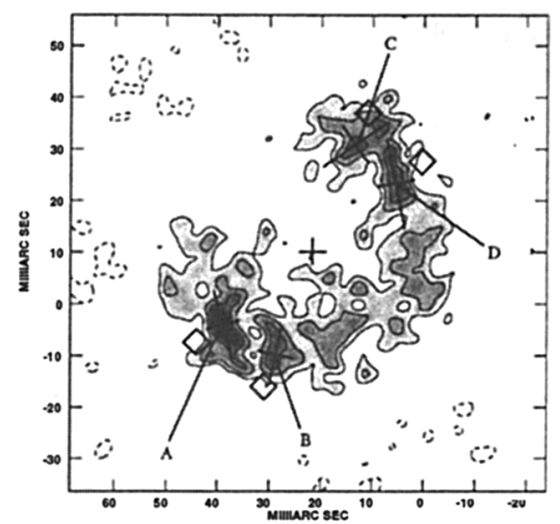

Figure 1. Global VLBI image of the young supernova remnant, $43.31+592$.

tioned young supernova remnant, $43.31+592$ and the most compact source in M82, 41.95 +575 (Muxlow et al. these proceedings).

\section{The Evolution of $43.31+592$}

The new global VLBI map of $43.31+592$ is shown in figure 1. Labels A, B, C and $D$ denote the four most significant knots of radio emission and the cross marks the average position of these knots. By comparing EVN maps of the source at epochs 1986 and 1997, an average expansion speed of $9500 \mathrm{~km} \mathrm{~s}^{-1}$ was measured by Pedlar et al. (1999). The diamonds in figure 1 show the predicted positions of the four labelled components ten years after the last observations assuming this expansion rate continues.

However, we also know that the remnant existed in 1972, as shown by the $8.4 \mathrm{GHz}$ map of Kronberg \& Wilkinson (1975). By parameterising the size evolution of the shell as $\mathrm{D}_{p c}=\mathrm{kT}_{y r}^{\delta}$ and using the measured shell diameter of $43.31+592$ at epochs 1986.7 and 1997.6 as $0.442 \pm 0.016$ and $0.653 \pm 0.023 \mathrm{pc}$ respectively, we derive a limit on the deceleration parameter of $\delta>0.73 \pm 0.11$.

\section{References}

Kronberg, P. P., \& Wilkinson, P. N. 1975, ApJ, 200, 430.

Kronberg, P. P., Biermann, P., Schwab, F. R. 1985, ApJ, 291, 693.

Muxlow, T. W. B., Pedlar, A., Wilkinson, P. N., Axon, D. J., Sanders, E. M., de Bruyn, A. G. 1994, MNRAS 266, 455.

Unger, S. W., Pedlar, A., Axon. D. J., Wilkinson, P. N., Appleton, P. N. 1984, MNRAS, 211, 783. 